\title{
Spinal cord injury and the human microbiome: beyond the brain-gut axis
}

\author{
David J. Wallace, MD, ${ }^{1}$ Naomi L. Sayre, PhD, ${ }^{1,2}$ T. Tyler Patterson, BA, ${ }^{3}$ \\ Susannah E. Nicholson, MD, ${ }^{4}$ Donald Hilton, MD, ${ }^{1}$ and Ramesh Grandhi, MD ${ }^{1,4,5}$
}

\begin{abstract}
'Department of Neurosurgery, ${ }^{3}$ Long School of Medicine, and ${ }^{4}$ Division of Trauma and Emergency Surgery, Department of Surgery, University of Texas Health Sciences Center at San Antonio; ${ }^{2}$ South Texas Veteran's Health Care System, Audie L. Murphy Division, San Antonio, Texas; and 'Department of Neurosurgery, University of Utah School of Medicine, Salt Lake City, Utah
\end{abstract}

\begin{abstract}
In addition to standard management for the treatment of the acute phase of spinal cord injury (SCI), implementation of novel neuroprotective interventions offers the potential for significant reductions in morbidity and long-term health costs. A better understanding of the systemic changes after $\mathrm{SCl}$ could provide insight into mechanisms that lead to secondary injury. An emerging area of research involves the complex interplay of the gut microbiome and the CNS, i.e., a brain-gut axis, or perhaps more appropriately, a CNS-gut axis. This review summarizes the relevant literature relating to the gut microbiome and SCl. Experimental models in stroke and traumatic brain injury demonstrate the bidirectional communication of the CNS to the gut with postinjury dysbiosis, gastrointestinal-associated lymphoid tissue-mediated neuroinflammatory responses, and bacterial-metabolite neurotransmission. Similar findings are being elucidated in SCl as well. Experimental interventions in these areas have shown promise in improving functional outcomes in animal models. This commensal relationship between the human body and its microbiome, particularly the gut microbiome, represents an exciting frontier in experimental medicine.
\end{abstract}

https://thejns.org/doi/abs/10.3171/2018.12.FOCUS18206

KEYWORDS microbiome; spinal cord injury; brain-gut axis

$\Lambda$ $\mathrm{T}$ the cellular level, spinal cord injury (SCI) is a polyphasic insult. ${ }^{43}$ First, direct mechanical injury results in damaged cell membranes, ischemia, and accumulated neurotransmitters. Secondary processes increase the size of the injury, causing cell death propagated by cascades of free radical production, energy failure, immune cell invasion, and glutamatergic excitotoxicity. In the chronic stages of SCI, astroglial scar formation, continued demyelination, syrinx formation, and other regenerative processes can continue for years at the cellular level.

It should be no surprise that the multiple injury phases involve systemic modifiers. At least a part of this process involves the brain-gut axis, the major bidirectional communication pathway between the brain and the gastrointestinal (GI) system that incorporates both afferent and efferent signals involving neuronal, hormonal, and immunological pathways. ${ }^{42}$ To date, most of the existing research following CNS injury pertaining to the micro- biome involves studying changes to the gut microbiome following stroke or traumatic brain injury (TBI). ${ }^{4,26,38,39,42 \text {, }}$ 48,49,52 Thus, there is a paucity of research involving SCI and the bidirectional influence on the gut microbiome. The purpose of this paper is to review the literature published on the microbiome and highlight the changes that occur following SCI. By developing an understanding of the role that the microbiome has in potentiating inflammation or protecting against secondary injury, it may be possible to enhance patient care, identify therapeutic targets for neuroprotection, and potentially improve outcomes.

\section{Brief History of the Human Microbiome}

Shortly after the Human Genome Project was completed, the Human Microbiome Project became the next frontier for understanding the human "supra-organism." 44 The microorganisms living within and on the human body

ABBREVIATIONS GALT = GI-associated lymphoid tissue; GI = gastrointestinal; IL = interleukin; MIP-2 = macrophage inflammatory protein 2; SCI = spinal cord injury; TBI $=$ traumatic brain injury; TNF- $\alpha=$ tumor necrosis factor-alpha. 
outnumber human cells, and symbiotic relationships are being explored in healthy and disease states. ${ }^{35}$ The microbiome is recognized as a source of genetic diversity, component of host immunity, and effector on nutrient and drug metabolism. ${ }^{15}$ Knowledge of the taxonomic and functional composition of the gut microbiome has grown exponentially with the use of 16s ribosomal RNA encoding and metagenomic technologies, which have replaced the previous generation of culture-based approaches. ${ }^{1,15}$ Early investigations into the microbiome and disease states were related to obesity and inflammatory bowel disease. ${ }^{24,45}$ Subsequently, non-GI correlations with pathological conditions were noted in cardiovascular disease, asthma, and even autism. ${ }^{12,19}$ As the field has progressed, the gut microbiome has been linked to essentially all organ systems.

\section{Microbiome and Injury}

The link between surgical critical illness, systemic inflammation, and pathological changes in gut bacteria is decades old. ${ }^{7,27}$ Trauma, burns, sepsis, and surgical injuries can result in a constellation of dysfunctions in intestinal transit, expression of nutrient transporters, and mucosal barrier changes. ${ }^{30}$ These alterations allow bacterial dysbiosis in the gut, which leads to the development of a systemic inflammatory response. Unsurprisingly, the maintenance of the integrity of the intestinal mucosal epithelium is multifactorial; gut bacteria may be the most important factor, along with intraluminal contents, pancreatic enzymes, hepatobiliary factors, and the enteric and central nervous system. ${ }^{13,30,36}$

An unstable microbiome during injury more closely resembles that of an infectious state. The intestinal microbiota becomes fundamentally disrupted within hours of injury, with quantitative reductions of 1000-fold compared to healthy controls. ${ }^{18}$ Furthermore, among patients with sepsis, the composition of the gut microbiome dramatically changes such that communities of ultra-lowdiversity pathogens emerge and trigger further virulence in the host. ${ }^{53}$ Krezalek and colleagues noted that these disturbances were predictive of sepsis-associated mortality in their patient population. ${ }^{25}$ Intestinal wall damage leads to inflammation of the mucosal barrier, which results in an altered GI tract with higher levels of nitrate and abnormal gradient of mucosal oxygen. ${ }^{9}$ As a result, the changes to the environment lead to a proliferation of Proteobacteria such as Pseudomonas aeruginosa and Escherichia coli, as well as certain Firmicutes including Staphylococcus aureus and Enterococcus species.

Burn injury is an obvious model for gut biome research given its prevalence and clinical observations that $75 \%$ of all deaths after severe burns are related to sepsis and infectious complications. ${ }^{8}$ Demonstrated in both human and murine models, burn injury alters the structure of the intestinal biome to promote gram-negative aerobic bacteria and leads to increased intestinal permeability to allow bacterial translocation to normally sterile sites..$^{10}$ Overall, healthy bacteria such as those in the Lachnospiraceae family are reduced, while Enterobacteriacea family flourish, species often found in septic patients. ${ }^{8,10}$ The integrity of the gut epithelium itself may have a fundamental role in the development of sepsis following burn injury. These findings support the concept that trauma in many forms causes disruption in the gut, which in turn acts in a pathological manner.

\section{The Feed-Forward Effect on the CNS-Gut Axis in Injury}

The use of stroke models to investigate the brain-gut axis has given interesting insight into a brain-gut feedforward loop. ${ }^{37}$ Murine large-volume stroke models demonstrated increased gut permeability and bacterial translocation associated with clinical poststroke infections. .,40 $^{4}$ In human patients, Yamashiro et al. found that ischemic stroke is associated with gut dysbiosis and systemic inflammation..$^{51}$

In contrast to stroke, TBI appears to offer independent disruptions to intestinal integrity. Clinically, TBI can result in chronic dysfunction of the GI system, and disability. ${ }^{22}$ Murine-TBI models have shown ultrastructural gut changes within 3 hours of injury; epithelial cell tight junction integrity is impaired, and tight junction proteins that normally maintain close cellular contacts (claudin and occludin) have decreased expression. ${ }^{17}$ Experimental blockades of these protein changes ameliorate post-TBI increases in gut permeability.,5 This diminished integrity and loss of tight junction protein expression may be caused by increased tumor necrosis factor-alpha (TNF- $\alpha$ ) and myosin light chain. ${ }^{32,34,46}$ However, Ma et al. found that moderate-level TBI in mice elicited intestinal histopathology independent of inflammation. ${ }^{26}$ They observed histopathological increases in mucosal depth and smooth muscle thickening, increased expression of glial fibrillary acidic protein and SOX10 expression (associated with enteric glial cell activation), and functional increases in paracelluar flux at 28 days postinjury.

One potential explanation for the ability of the brain to affect gut integrity independent of inflammation involves the enteric nervous system and neuroendocrine signals. ${ }^{13,21,30}$ One part of the enteric nervous system, vagal motor activity, has been shown to be impaired after TBI, and intervention via closed-loop stimulation has shown improvement in functional recovery in two different murine studies. ${ }^{2,31}$ Such results show promise in elucidating the CNS-gut axis and its role in secondary processes after brain injury.

New investigations are now underway that examine the relationship of microbiome and SCI, particularly the concept that the brain-gut axis may be a component of a larger CNS-microbiota-gut axis. Kabatas et al. demonstrated temporal GI changes after SCI in a rat model. ${ }^{20}$ They found decreased gastric emptying and slowed GI transit 4 weeks after SCI. Notably, they were unable to show such changes in the acute phase (within 48 hours). This delay was linked to neurotransmission, as demonstrated by slowing of the inhibitory junction potentials regulated by decreases in GI vasoactive intestinal polypeptide and neuronal nitric oxide synthase. Lastly, they demonstrated histopathological thinning of the GI mucosa and muscle layers at 4 weeks postinjury.

O'Connor et al. used a rat SCI model to investigate 
microbiome and proinflammatory cytokine changes. ${ }^{29}$ At 8 weeks postinjury, their SCI group showed significantly higher levels of Bifidobacteriaceae and Turibacteraceae families, with depletion of Clostridium saccharogumia species compared to controls. The analysis of concomitant proinflammatory cytokines found that interleukin (IL)-1 $\beta$, IL-12, and macrophage inflammatory protein 2 (MIP-2) were significantly correlated with microbiome changes in animals with SCI. MIP-2 and TNF- $\alpha$ were significantly higher at the 4-week analysis than at the 8-week analysis after SCI.

Kigerl et al. made similar observations. ${ }^{23}$ These investigators demonstrated gut dysbiosis occurs with SCI, and that premorbid dysbiosis leads to poorer neurological outcomes and neuropathological findings postinjury. Analysis of proinflammatory cytokines also found increased expression of TNF, IL-1 $\beta$, and IL-10 in the mesenteric lymph nodes by postinjury day 7. GI-associated lymphoid tissue (GALT) inflammatory response was marked by increases in B lymphocytes, CD8-positive T lymphocytes, dendritic cells, and macrophages with reductions in CD4-positive T lymphocytes. Clinical investigation into human gut dysbiosis after SCI seems warranted based on these laboratory findings in animal models.

\section{The Feedback Effect on the Brain-Gut Axis in CNS Injury}

The more surprising feedback effect of the gut on the CNS has been demonstrated in several experiments. Ma et al. found mice subjected to a bacterial-diarrhea infection model 4 weeks after TBI showed increased astrocyte and microglia inflammation with corresponding increases in brain lesion sizes. ${ }^{26}$ Our group has recently shown that significant changes in the gut microbiome are evident within 2 hours following a moderate TBI induced in rats. A decrease in the relative abundance of traditionally beneficial bacteria was observed, specifically among bacteria within the Firmicutes phylum, with concomitant increases in families that contained pathogenic bacteria including Bacteroidaceae in the phylum Bacteroidetes and Enterobacteriaceae and Pseudomonadaceae in the phylum Proteobacteria. Moreover, the changes observed in the bacterial diversity correlated with post-TBI, MRI-determined lesion volume and loss of behavioral function in the animal model..$^{28}$ Two laboratories have independently demonstrated the brain-gut pathway after stroke, which affects gut dysbiosis, neuroinflammatory injury response, and functional outcomes. ${ }^{4,39}$ Singh et al. showed germ-free mice recolonized with poststroke microbiota had larger stroke lesions. Conversely, Benakis et al. used antibiotics to alter the composition of the microbiota to reduce infarct volumes in mice subjected to transient middle cerebral artery occlusion. Both groups found benefit of therapeutic fecal transplantation on stroke lesions and functional outcomes. Certain probiotic treatments were neuroprotective in murine stroke models. ${ }^{41}$ These experiments demonstrated immune cell-dependent mechanisms as evident by intestinal IL-10, IL-17, regulatory T cells, T-helper, and $\gamma \delta$ T-cell changes in intestinal and CNS tissues correlating with stroke lesions and functional outcomes (Fig. 1).
The first study examining this link in SCI was by Kigerl et al. ${ }^{23}$ Antibiotic-induced dysbiotic mice showed more severe SCI lesions; they showed that resuscitation with probiotics improved postinjury recovery. One potential mechanism opined by the authors involved reductions in the amount of available neuroactive bacterial metabolites, including serotonin precursors and short-chain fatty acids. This comes from work that has shown these metabolites can bypass the blood-brain barrier to influence CNS structure and function and induce T-cell differentiation in the gut, which plays a role in the CNS injury response. ${ }^{11,14,33,47}$

Taken together, these findings indicate that SCI elicits a dysbiosis response in the gut, and suggests the gut, in turn, can modulate the neuroinflammatory response in the CNS. Given the influence of the composition of the gut microbiome on the homeostasis of the CNS, the interplay of these bacterial populations in digestion, immunity, disease, and health is of great interest. Developing further understanding of this interplay in healthy and pathological conditions following CNS injury may prove invaluable.

\section{Limitations}

In microbiome research, it is paramount to closely examine causation and mechanisms over correlation and bystander effects..$^{16}$ Additionally, germ-free or antibiotic-treated mice have often been used as controls, raising important questions as to the validity of these models for comparison. As is the case in most animal research, consideration must be paid to rodent and human differences including anatomy, diet, baseline/healthy microbiota, immunology, comorbidities, and social dynamics. ${ }^{49}$ Although some laboratory successes have been published, translational trials have tempered enthusiasm. Studies into the benefit of probiotics in TBI remain inconclusive. ${ }^{6}$ Fecal microbiota transplantation has been attempted for Parkinson's disease, multiple sclerosis, autism, and chronic fatigue syndrome, with little explanation of therapeutic mechanism. ${ }^{50}$

Future studies investigating the temporal changes in the microbiome after SCI could offer insight into the many comorbid conditions faced by patients years after SCI. Changes in neuroenteric innervation, gut mobility, neurogenic bladder, and even locomotion may lead to microbiome changes that predispose patients with chronic SCI to infections including septicemia, urinary tract infections, $C$. difficile colitis, and decubitus ulcers.

\section{Conclusions}

Changes in the gut microbiome have been demonstrated in a wide variety of injury models and appear to be affected by an array of local and systemic phenomena. Bacterial translocation and sepsis following polytrauma and burn injuries illustrates the impact of the microbiome following systemic injuries. A similar "pathobiome" can be seen following CNS injury, with the potential for targeted interventions to mitigate secondary damage via the braingut axis and altering the neuroinflammatory feedback loop. Acute phase changes including bacterial metabolites, intestinal permeability, and GALT-associated immunity warrant further exploration as intervention points in SCI. Crossover applicability in treatments for SCI, TBI, and 


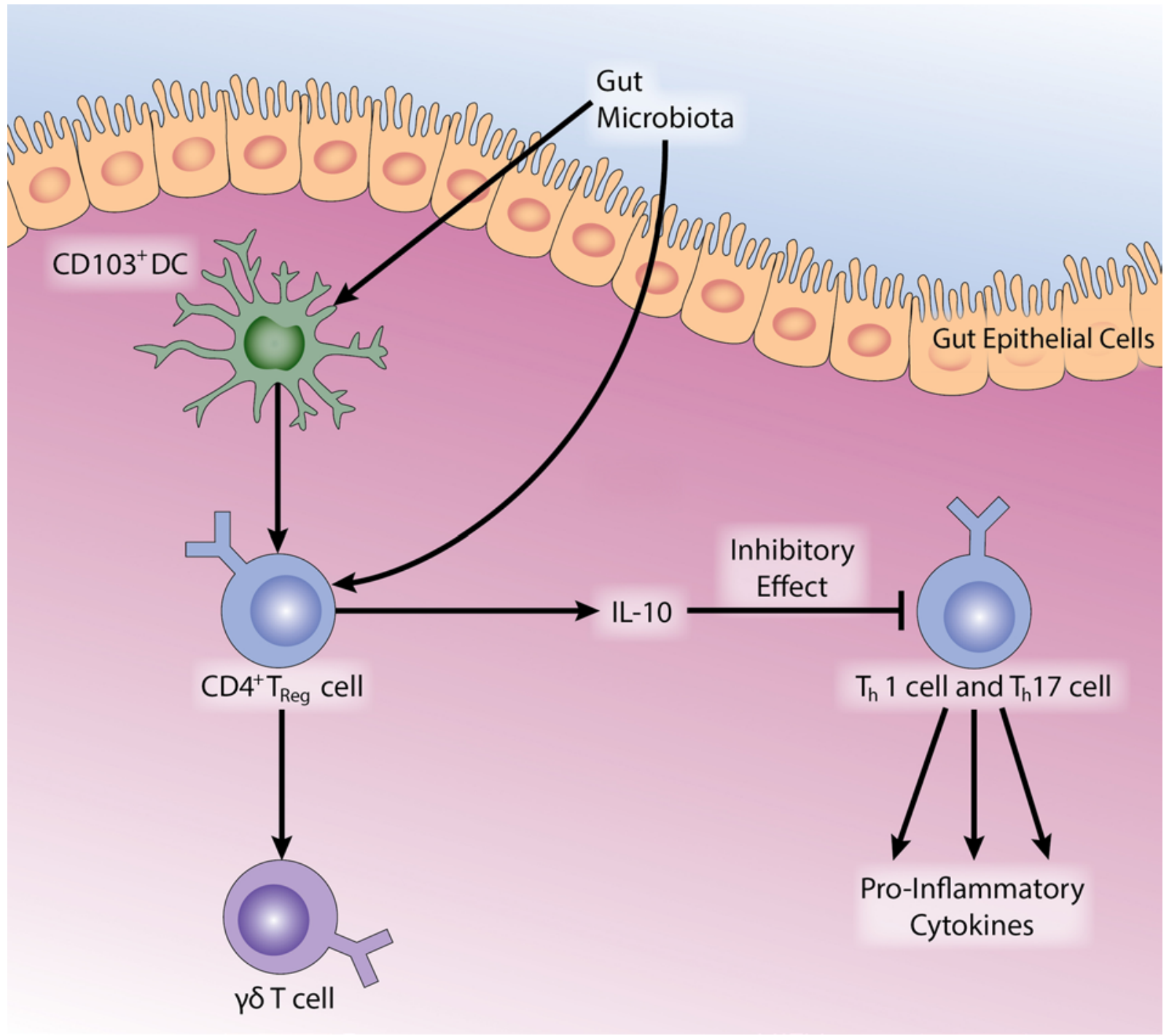

FIG. 1. CD4-positive (CD4+) regulatory $T$ cells $\left(T_{\text {reg }}\right)$ serve as a fulcrum in the balance of proinflammatory and antiinflammatory immune modulation. In the gut, microbiota are sampled or may translocate, resulting in an immune response. The CD103-positive dendritic cells $\left(C D 103^{+} D C\right)$, a type of antigen-presenting cell of the intestinal mucosa, have been found to drive the proliferation of $T_{\text {reg }}$ cells while the microbiota is in a steady state. Subsequent activity by $T_{\text {reg }}$ cells results in suppression of an inflammatory response by multiple mechanisms. A central focus of the current stroke research is the $T_{\text {reg }}$ cell-mediated influence on $\gamma \delta T$ cells. $\gamma \delta$ T cells have the potential to cause either inflammatory or antiinflammatory effects, but the influence of $\mathrm{T}_{\text {reg }}$ cells while gut microbiota remain in a steady state urges a reduced inflammatory state. $\mathrm{T}_{\text {reg }}$ cells additionally produce IL-10, further suppressing $T$ helper $\left(T_{h}\right) 1$ and $T_{h} 17$ responses.

stroke via the gut microbiome is possible. Enthusiasm for new applications of this science must be tempered while mechanisms are elucidated to exclude correlation and bystander effects.

\section{References}

1. Arumugam M, Raes J, Pelletier E, Le Paslier D, Yamada T, Mende DR, et al: Enterotypes of the human gut microbiome. Nature 473:174-180, 2011

2. Bansal V, Costantini T, Ryu SY, Peterson C, Loomis W, Putnam J, et al: Stimulating the central nervous system to prevent intestinal dysfunction after traumatic brain injury. J Trauma 68:1059-1064, 2010

3. Bansal V, Ryu SY, Blow C, Costantini T, Loomis W, Eliceiri
B, et al: The hormone ghrelin prevents traumatic brain injury induced intestinal dysfunction. J Neurotrauma 27:22552260, 2010

4. Benakis C, Brea D, Caballero S, Faraco G, Moore J, Murphy $\mathrm{M}$, et al: Commensal microbiota affects ischemic stroke outcome by regulating intestinal $\gamma \delta$ T cells. Nat Med 22:516523, 2016

5. Beynon AL, Brown MR, Wright R, Rees MI, Sheldon IM, Davies JS: Ghrelin inhibits LPS-induced release of IL-6 from mouse dopaminergic neurones. J Neuroinflammation 10:40, 2013

6. Brenner LA, Stearns-Yoder KA, Hoffberg AS, Penzenik ME, Starosta AJ, Hernández TD, et al: Growing literature but limited evidence: a systematic review regarding prebiotic and probiotic interventions for those with traumatic brain injury 
and/or posttraumatic stress disorder. Brain Behav Immun 65:57-67, 2017

7. Carrico CJ, Meakins JL, Marshall JC, Fry D, Maier RV: Multiple-organ-failure syndrome. Arch Surg 121:196-208, 1986

8. Church D, Elsayed S, Reid O, Winston B, Lindsay R: Burn wound infections. Clin Microbiol Rev 19:403-434, 2006

9. Dickson RP: The microbiome and critical illness. Lancet Respir Med 4:59-72, 2016

10. Earley ZM, Akhtar S, Green SJ, Naqib A, Khan O, Cannon $\mathrm{AR}$, et al: Burn injury alters the intestinal microbiome and increases gut permeability and bacterial translocation. PLoS One 10:e0129996, 2015

11. El Aidy S, Dinan TG, Cryan JF: Gut Microbiota: The conductor in the orchestra of immune-neuroendocrine communication. Clin Ther 37:954-967, 2015

12. Finegold SM: Therapy and epidemiology of autism-clostridial spores as key elements. Med Hypotheses 70:508-511, 2008

13. Furness JB, Callaghan BP, Rivera LR, Cho HJ: The enteric nervous system and gastrointestinal innervation: integrated local and central control. Adv Exp Med Biol 817:39-71, 2014

14. Furusawa Y, Obata Y, Fukuda S, Endo TA, Nakato G, Takahashi D, et al: Commensal microbe-derived butyrate induces the differentiation of colonic regulatory T cells. Nature 504:446-450, 2013

15. Grice EA, Segre JA: The human microbiome: our second genome. Annu Rev Genomics Hum Genet 13:151-170, 2012

16. Hanage WP: Microbiology: Microbiome science needs a healthy dose of scepticism. Nature 512:247-248, 2014

17. Hang CH, Shi JX, Li JS, Wu W, Y in HX: Alterations of intestinal mucosa structure and barrier function following traumatic brain injury in rats. World J Gastroenterol 9:2776-2781, 2003

18. Hayakawa M, Asahara T, Henzan N, Murakami H, Yamamoto H, Mukai N, et al: Dramatic changes of the gut flora immediately after severe and sudden insults. Dig Dis Sci 56:2361-2365, 2011

19. Holmes E, Loo RL, Stamler J, Bictash M, Yap IKS, Chan Q, et al: Human metabolic phenotype diversity and its association with diet and blood pressure. Nature 453:396-400, 2008

20. Kabatas S, Yu D, He XD, Thatte HS, Benedict D, Hepgul KT, et al: Neural and anatomical abnormalities of the gastrointestinal system resulting from contusion spinal cord injury. Neuroscience 154:1627-1638, 2008

21. Katzenberger RJ, Ganetzky B, Wassarman DA: The gut reaction to traumatic brain injury. Fly (Austin) 9:68-74, 2015

22. Kharrazian D: Traumatic brain injury and the effect on the brain-gut axis. Altern Ther Health Med 21 (Suppl 3):2832,2015

23. Kigerl KA, Hall JCE, Wang L, Mo X, Yu Z, Popovich PG: Gut dysbiosis impairs recovery after spinal cord injury. J Exp Med 213:2603-2620, 2016

24. Kinross JM, Darzi AW, Nicholson JK: Gut microbiome-host interactions in health and disease. Genome Med 3:14, 2011

25. Krezalek MA, DeFazio J, Zaborina O, Zaborin A, Alverdy JC: The shift of an intestinal "microbiome" to a "pathobiome" governs the course and outcome of sepsis following surgical injury. Shock 45:475-482, 2016

26. Ma EL, Smith AD, Desai N, Cheung L, Hanscom M, Stoica $\mathrm{BA}$, et al: Bidirectional brain-gut interactions and chronic pathological changes after traumatic brain injury in mice. Brain Behav Immun 66:56-69, 2017

27. Marshall JC, Christou NV, Meakins JL: The gastrointestinal tract. The "undrained abscess" of multiple organ failure. Ann Surg 218:111-119, 1993

28. Nicholson SE, Watts LT, Burmeister DM, Merrill D, Scrog- gins S, Zou Y, et al: Moderate traumatic brain injury alters the gastrointestinal microbiome in a time-dependent manner. Shock [epub ahead of print], 2018

29. O'Connor G, Jeffrey E, Madorma D, Marcillo A, Abreu MT, Deo SK, et al: Investigation of microbiota alterations and intestinal inflammation post-spinal cord injury in rat model. J Neurotrauma 35:2159-2166, 2018

30. Patel JJ, Rosenthal MD, Miller KR, Martindale RG: The gut in trauma. Curr Opin Crit Care 22:339-346, 2016

31. Pruitt DT, Schmid AN, Kim LJ, Abe CM, Trieu JL, Choua $\mathrm{C}$, et al: Vagus nerve stimulation delivered with motor training enhances recovery of function after traumatic brain injury. J Neurotrauma 33:871-879, 2016

32. Ringel Y, Maharshak N: Intestinal microbiota and immune function in the pathogenesis of irritable bowel syndrome. Am J Physiol Gastrointest Liver Physiol 305:G529-G541, 2013

33. Rothhammer V, Mascanfroni ID, Bunse L, Takenaka MC, Kenison JE, Mayo L, et al: Type I interferons and microbial metabolites of tryptophan modulate astrocyte activity and central nervous system inflammation via the aryl hydrocarbon receptor. Nat Med 22:586-597, 2016

34. Saulnier DM, Ringel Y, Heyman MB, Foster JA, Bercik P, Shulman RJ, et al: The intestinal microbiome, probiotics and prebiotics in neurogastroenterology. Gut Microbes 4:17-27, 2013

35. Sender R, Fuchs S, Milo R: Are we really vastly outnumbered? Revisiting the ratio of bacterial to host cells in humans. Cell 164:337-340, 2016

36. Sertaridou E, Papaioannou V, Kolios G, Pneumatikos I: Gut failure in critical care: old school versus new school. Ann Gastroenterol 28:309-322, 2015

37. Sherwin E, Dinan TG, Cryan JF: Recent developments in understanding the role of the gut microbiota in brain health and disease. Ann N Y Acad Sci 1420:5-25, 2018

38. Simon DW, McGeachy MJ, Bayır H, Clark RSB, Loane DJ, Kochanek PM: The far-reaching scope of neuroinflammation after traumatic brain injury. Nat Rev Neurol 13:572, 2017

39. Singh V, Roth S, Llovera G, Sadler R, Garzetti D, Stecher B, et al: Microbiota dysbiosis controls the neuroinflammatory response after stroke. J Neurosci 36:7428-7440, 2016

40. Stanley D, Mason LJ, Mackin KE, Srikhanta YN, Lyras D, Prakash MD, et al: Translocation and dissemination of commensal bacteria in post-stroke infection. Nat Med 22:12771284,2016

41. Sun J, Ling Z, Wang F, Chen W, Li H, Jin J, et al: Clostridium butyricum pretreatment attenuates cerebral ischemia/ reperfusion injury in mice via anti-oxidation and anti-apoptosis. Neurosci Lett 613:30-35, 2016

42. Sundman MH, Chen NK, Subbian V, Chou YH: The bidirectional gut-brain-microbiota axis as a potential nexus between traumatic brain injury, inflammation, and disease. Brain Behav Immun 66:31-44, 2017

43. Thuret S, Moon LDF, Gage FH: Therapeutic interventions after spinal cord injury. Nat Rev Neurosci 7:628-643, 2006

44. Turnbaugh PJ, Ley RE, Hamady M, Fraser-Liggett CM, Knight R, Gordon JI: The human microbiome project. Nature 449:804-810, 2007

45. Turnbaugh PJ, Ley RE, Mahowald MA, Magrini V, Mardis ER, Gordon JI: An obesity-associated gut microbiome with increased capacity for energy harvest. Nature 444:10271031,2006

46. Turner JR: Intestinal mucosal barrier function in health and disease. Nat Rev Immunol 9:799-809, 2009

47. Wikoff WR, Anfora AT, Liu J, Schultz PG, Lesley SA, Peters EC, et al: Metabolomics analysis reveals large effects of gut microflora on mammalian blood metabolites. Proc Natl Acad Sci U S A 106:3698-3703, 2009

48. Winek K, Engel O, Koduah P, Heimesaat MM, Fischer A, 
Bereswill S, et al: Depletion of cultivatable gut microbiota by broad-spectrum antibiotic pretreatment worsens outcome after murine stroke. Stroke 47:1354-1363, 2016

49. Winek K, Meisel A, Dirnagl U: Gut microbiota impact on stroke outcome: fad or fact? J Cereb Blood Flow Metab 36:891-898, 2016

50. Xu MQ, Cao HL, Wang WQ, Wang S, Cao XC, Yan F, et al: Fecal microbiota transplantation broadening its application beyond intestinal disorders. World J Gastroenterol 21:102111,2015

51. Yamashiro K, Tanaka R, Urabe T, Ueno Y, Yamashiro Y, Nomoto K, et al: Gut dysbiosis is associated with metabolism and systemic inflammation in patients with ischemic stroke. PLoS One 12:e0171521, 2017

52. Yin J, Liao SX, He Y, Wang S, Xia GH, Liu FT, et al: Dysbiosis of gut microbiota with reduced trimethylamine-Noxide level in patients with large-artery atherosclerotic stroke or transient ischemic attack. J Am Heart Assoc 4:e002699, 2015

53. Zaborin A, Smith D, Garfield K, Quensen J, Shakhsheer B, Kade M, et al: Membership and behavior of ultra-low-diversity pathogen communities present in the gut of humans during prolonged critical illness. MBio 5:e01361-14, 2014

\section{Disclosures}

The authors report no conflict of interest concerning the materials or methods used in this study or the findings specified in this paper.

\section{Author Contributions}

Conception and design: Wallace, Sayre, Patterson, Nicholson, Grandhi. Analysis and interpretation of data: Grandhi. Drafting the article: all authors. Critically revising the article: all authors. Reviewed submitted version of manuscript: Wallace, Hilton, Grandhi. Approved the final version of the manuscript on behalf of all authors: Wallace. Study supervision: Sayre, Hilton, Grandhi.

\section{Correspondence}

David J. Wallace: University of Texas Health Sciences Center at San Antonio, San Antonio, TX. wallaced2@uthscsa.edu. 